\title{
The Association Between Obesity and the Frailty Syndrome in Older Women: The Women's Health and Aging Studies
}

\author{
Caroline S. Blaum, MD, MS, ${ }^{* \dagger}$ Qian Li Xue, PhD, ${ }^{\ddagger}$ Elisabete Michelon, MD, ${ }^{\mathcal{S}}$ Richard D. Semba, MD, \\ $M P H, \stackrel{\ddagger}{\ddagger}$ and Linda P. Fried, MD, MPH ${ }^{\ddagger}$
}

(See editorial comments by Dr. Kenneth Rockwood on pp 1069-1070)

OBJECTIVES: To determine whether obesity is associated with the frailty phenotype and, if so, whether comorbid conditions or inflammatory markers explain this association.

DESIGN: Cross-sectional analysis of baseline data from the Women's Health and Aging Studies I (1992) and II (1994), complementary population-based studies.

SETTING: Twelve contiguous ZIP code areas in Baltimore, Maryland.

PARTICIPANTS: Five hundred ninety-nine communitydwelling women aged 70 to 79 with a body mass index (BMI) greater than $18.5 \mathrm{~kg} / \mathrm{m}^{2}$.

MEASUREMENTS: The dependent variables were the frailty syndrome, including prefrailty, defined as presence of one or two of five frailty indicators (weakness, slowness, weight loss, low physical activity, exhaustion), and frailty, defined as three or more indicators. Independent variables included BMI, categorized using World Health Organization criteria as normal $\left(18.5\right.$ to $\left.<25 \mathrm{~kg} / \mathrm{m}^{2}\right)$, overweight ( 25 to $\left.<30 \mathrm{~kg} / \mathrm{m}^{2}\right)$, and obese $\left(\geq 30 \mathrm{~kg} / \mathrm{m}^{2}\right)$; chronic diseases; $\mathrm{C}$-reactive protein; and serum carotenoids.

RESULTS: Being overweight was significantly associated with prefrailty, and obesity was associated with prefrailty and frailty. In all frail women, regardless of BMI group, a similar pattern of three defining frailty indicators was

From the *University of Michigan; ${ }^{\dagger}$ Ann Arbor Veterans Affairs Healthcare System Geriatric Rehabilitation, Education and Clinical Center, Ann Arbor, Michigan; Johns Hopkins Medical Institutions, Baltimore, Maryland; ${ }^{\S}$ Institute of Geriatrics and Gerontology, Pontifical Catholic University of Rio Grande do Sul, Rio Grande do Sul, Brazil.

This work was supported by National Institute on Aging Grant RO1 AG11703-01A1, National Institutes of Health, National Center for Research Resources, General Clinical Research Center Grants RR00722 and RO1 AI41956, and National Institute on Aging Contract NO1-AG12112. Dr Blaum was supported by NIA-K08 AG00749-02 and the Ann Arbor Veterans Affairs Geriatric Research, Education and Clinical Center. Dr. Michelon was supported by a postdoctoral scholarship from CNPq-Brazil.

Address correspondence to Caroline S. Blaum, MD, MS, Associate Professor, The University of Michigan Medical School, CCGCB Room 1111, 1500 East Medical Center Drive, Ann Arbor, MI 48109. E-mail: cblaum@umich.edu

DOI: $10.1111 /$ j.1532-5415.2005.53300.x found: slowness, weakness, and low activity (with the addition of weight loss in the normal weight group.) In multinomial regression models, obesity was significantly associated with prefrailty (odds ratio $(\mathrm{OR})=2.23,95 \%$ confidence interval $(\mathrm{CI})=1.29-3.84)$ and frailty $(\mathrm{OR}=$ $3.52,95 \% \mathrm{CI}=1.34-9.13$ ), even when controlling for covariates.

CONCLUSION: Obesity is associated with the frailty syndrome in older women in cross-sectional data. This association remains significant even when multiple conditions associated with frailty are considered. Prospective studies are needed to confirm this finding. J Am Geriatr Soc 53:927-934, 2005.

Key words: overweight and obese; frailty; older women

$T$ he geriatric syndrome of frailty is a syndrome of physical vulnerability characterized by multisystem dysfunction and lack of physiological reserve. ${ }^{1-4}$ It is associated with medical comorbidities and with increased risk of disability, increased health services usage, and mortality. ${ }^{5,6}$ An empirical definition of the frailty phenotype, which includes weight loss, slowness, exhaustion, poor exercise tolerance, and weakness, has been proposed. ${ }^{3}$ Three of these frailty indicators were considered to define the full frailty syndrome, whereas the presence of one or two was considered prefrail syndrome, associated with increased risk of frailty. Using this definition, it was demonstrated that older adults with frailty had a greater prevalence of multiple diseases, including heart failure, atherosclerotic diseases, and diabetes mellitus.

Although obesity is well known to be associated with disability, ${ }^{7}$ its association with the frailty syndrome is less obvious, particularly because frailty is considered a wasting disorder, ${ }^{8,9}$ and weight loss is a possible but not necessary component of the syndrome of frailty. Additionally, there is concern about confounding, because being overweight may directly cause slowness and poor exercise tolerance. However, there are several reasons to hypothesize a physiolog- 
ically based association between obesity and frailty. First, several researchers have described a distinct syndrome of "sarcopenic obesity" ${ }^{10-13}$ in obese people with a mismatch between fat and muscle. This syndrome is known to be strongly associated with decreased strength and increased mobility disability. ${ }^{10}$ Second, biochemical markers associated with frailty are higher in overweight people, particularly inflammatory markers such as C-reactive protein (CRP) and interleukin-6. ${ }^{14}$ Recent information has also shown that overweight/obese people have low carotenoids. ${ }^{15}$ Because of these associations, a syndrome of "obese frailty" can be hypothesized, although there have been few empirical data available to test this hypothesis. In a preliminary description of some physiological parameters associated with the frailty phenotype, an unexpectedly higher BMI was noted in frail older adults than in those who were not frail or were prefrail. ${ }^{16}$

The goal of the current research was to investigate potential cross-sectional associations between the frailty syndrome and obesity in older women. The frailty syndrome was defined using criteria defined empirically in the Cardiovascular Health Study (CHS) that were recently proposed. $^{3,16,17}$ These criteria were applied in baseline data from the Women's Health and Aging Studies (WHAS) I ${ }^{18,19}$ and II, ${ }^{20}$ complementary population-based epidemiological studies of older women in Baltimore which included the measures needed to define the frailty syndrome and study its association with obesity. Two hypotheses were evaluated: that obesity in older women is associated with the frailty syndrome and that the association between obesity and comorbid conditions and increased inflammation previously shown to be associated with frailty would largely explain this relationship. ${ }^{3,17}$

\section{METHODS}

\section{Study Design}

Cross-sectional secondary data analysis of the baseline rounds of related, population-based studies of older women was performed.

\section{Study Participants}

Participants in this study were women aged 70 to 79 who participated in WHAS I and II, two complementary, population-based studies designed to evaluate the causes and course of physical disability in older women living in the community. ${ }^{18-20}$ WHAS I consisted of an age-stratified random sample of women aged 65 and older selected from Medicare enrollees residing in 12 contiguous ZIP code areas in Baltimore. ${ }^{21}$ Eligible individuals were screened to identify self-reported physical disability, and WHAS I enrolled the one-third most-disabled women aged 65 and older. Of the 1,409 women who met study eligibility criteria, 1,002 agreed to participate in the study in 1992 to 1994. Standardized questionnaires, physical performance measures, and directed physical examination were administered in the participants' homes. Approximately $75 \%$ of the women also consented to phlebotomy.

WHAS II, begun in 1994, was specifically designed to be a companion study for WHAS I and includes a cohort of women aged 70 to 79 selected to be representative of the two-thirds least-disabled women living in the community. Participants $(n=436)$ were selected from age-stratified random samples from the same sampling frame as in WHAS I and were screened using the same methods. An interview, physical performance measures and directed physical examination standardized to that performed in WHAS I was administered in the Johns Hopkins Functional Status Laboratory. Phlebotomy was performed in $93 \%$ of WHAS II participants following the same protocol as that used in WHAS I. Details on the study methods and sampling design of the WHAS studies have been previously published. ${ }^{20,21}$

Analyses used a combined sample linking the two WHAS studies using a methodology that the WHAS research team developed. ${ }^{22}$ The sample consists of women participating in WHAS I or WHAS II who were aged 70 to 79 (WHAS I, $\mathrm{n}=399$; WHAS II, $\mathrm{n}=430, \mathrm{~N}=829$ ). Of these, 732 had at least one blood draw, although for the analyses, women were selected who had had blood drawn within 90 days of the baseline examination and a normal or higher BMI according to World Health Organization (WHO) criteria (rationale discussed in detail below, $\mathrm{n}=599,72 \%$ ). Weights have been calculated to account for sampling probability and response rates. This pooled sample has been used in published studies of the association between mobility disability and insulin-like growth factor levels and between serum carotenoids and sarcopenia. ${ }^{15,22}$

\section{Variables and Their Measurement \\ Defining Frailty: Application of the CHS Frailty Phenotype in WHAS}

The empirical measure of the prefrail/frailty syndrome is a composite variable recently described in $\mathrm{CHS}$ based on five indicators: weight loss, weakness, exhaustion, slowness, and low physical activity. ${ }^{3}$ The frailty phenotype is considered present if three or more of the indicators are present; the presence of one or two indicates a prefrail state. The five indicators of the frailty syndrome can also be studied individually.

Although the original measures were developed in the CHS study, similar or identical measures are present in WHAS I and II. The CHS phenotype ${ }^{3}$ was applied to the WHAS sample at baseline as closely as possible, given the measures available in WHAS. The following description will begin with the most comparable measure and proceed to the least comparable. Table 1 compares the WHAS frailty criteria measures with those of the CHS.

Grip strength was measured in WHAS according to the CHS protocol: level of maximal grip strength in the stronger hand. Speed in WHAS was based on a 4-m measured walk at usual pace. The subject could use a walking aid, but not the aid of another person. The slowness criterion merely rescaled the CHS criterion (15 feet) to apply to distance measured in WHAS. To measure energy expenditure, WHAS used a subset of the Minnesota Leisure Time Activities Questionnaire used in CHS, ${ }^{23}$ which was condensed from the original 18 activities to assess participation in six: walking, doing strenuous household chores, doing strenuous outdoor chores, dancing, bowling, and exercise. ${ }^{24}$ The WHAS threshold for defining low energy expenditure was therefore set at one-third of the CHS kcal threshold. 
Table 1. Frailty Defining Criteria: Women's Health and Aging Studies (WHAS) and Cardiovascular Health Study (CHS)

Characteristic

Weight loss

Exhaustion

Slowness

Low activity level

Weakness
WHAS

$\mathrm{BMI}<18.5$ or Weight at age 60 minus

weight at exam $\geq 10 \%$ of weight at age 60

Any of:

Low usual energy level $(\leq 3)$

Felt unusually tired in last month

Felt unusually weak in last month

Walking $4 \mathrm{~m}$ (speed) in:

$\leq 0.65 \mathrm{~m} / \mathrm{s}$ for height $\leq 159 \mathrm{~cm}$

$\leq 0.76 \mathrm{~m} / \mathrm{s}$ for height $>159 \mathrm{~cm}$

$<90 \mathrm{kcal}$ of physical expenditure on activity scale (6 items*)

Grip strength of the dominant hand:

$\leq 17 \mathrm{~kg}$ for $\mathrm{BMI} \leq 23$

$\leq 17.3 \mathrm{~kg}$ for $23<\mathrm{BMI} 26$

$\leq 18 \mathrm{~kg}$ for $26<\mathrm{BMI} 29$

$\leq 21 \mathrm{~kg}$ for $\mathrm{BMI}>29$
$\mathrm{CHS}$

Lost $>10$ pounds unintentionally in last year

Either of:

Felt that everything I did was an effort in last week

Could not get going in last week

Walking 15 feet (time) in:

$\leq 7$ seconds for height $\leq 159 \mathrm{~cm}$

$\leq 6$ seconds for height $>159 \mathrm{~cm}$

$<270 \mathrm{kcal}$ of physical expenditure on

activity scale (18 items ${ }^{\dagger}$ )

Grip strength of the dominant hand:

$\leq 17 \mathrm{~kg}$ for $\mathrm{BMI} \leq 23$

$\leq 17.3 \mathrm{~kg}$ for $23<\mathrm{BMI} \leq 26$

$\leq 18 \mathrm{~kg}$ for $26<\mathrm{BMI} \leq 29$

$\leq 21 \mathrm{~kg}$ for $\mathrm{BMI}>29$

\footnotetext{
* Walking for exercise, moderately strenuous household chores, moderately strenuous outdoor chores, bowling, regular exercise, dancing.

${ }^{\dagger}$ Walking for exercise, moderately strenuous household chores, mowing the lawn, raking the lawn, gardening, hiking, jogging, biking, exercise cycle, dancing, aerobics, bowling, golf, singles tennis, doubles tennis, racquetball, calisthenics, swimming.

$\mathrm{BMI}=$ body mass index; exam $=$ examination.
}

Although this choice has limitations, there was no obvious alternative.

Questions relating to exhaustion differed in the two studies, as summarized in Table 1. Exhaustion in WHAS was defined as an indicative response to at least one of three relevant questions: felt unusually tired in the previous month, felt unusually weak in the previous month, or had an unusually low energy level.

Weight loss is arguably the criterion that differs most across the studies. In CHS, participants were asked whether they had unintentionally lost more than 10 pounds in the previous year. This question was not asked at baseline of WHAS participants; rather, weight at age 60 was self-reported. (Future rounds of the WHAS studies ask about unintentional weight loss.) Frailty-eligible weight-loss criteria were applied if a woman's weight as measured at baseline represented a decrease of at least $10 \%$ relative to weight at age 60 or if a women's baseline measured BMI was less than $18.5 \mathrm{~kg} / \mathrm{m}^{2}$, the lowest WHO BMI risk category. Only 27 women in this sample had a BMI less than $18.5 \mathrm{~kg} / \mathrm{m}^{2}$; these women therefore had one frailty criterion by definition, and all had at least two more criteria and were thus frail. Because of the confounding by definition in this group, these 27 women were dropped from further analyses.

\section{BMI Categorization}

The research staff measured weight and height according to standardized protocols (these protocols were identical in WHAS I and 2) and BMI was calculated. BMI was categorized according to the WHO criteria as normal (18.5 to $<25 \mathrm{~kg} / \mathrm{m}^{2}$ ), overweight $\left(25\right.$ to $<30 \mathrm{~kg} / \mathrm{m}^{2}$ ), and obese $\left(\geq 30 \mathrm{~kg} / \mathrm{m}^{2}\right) .25,26$

\section{Comorbid Diseases}

WHAS I and II determined the prevalence of the major chronic diseases of aging, including heart failure, coronary artery disease, and diabetes mellitus, in older women at baseline. WHAS investigators adjudicated these diseases based on the questionnaire, physical examination measures, physician contact, and medical records. ${ }^{27}$ At the interview, a history of physician disease diagnoses was obtained. This information was validated using a combination of information obtained at the examination (medications used, symptoms, signs) and from the women's personal physicians and medical records. Qualified, trained clinicians then adjudicated the presence of disease according to a decision algorithm. Seventeen diseases have been ascertained; the methodology and algorithms have been published. ${ }^{27}$

\section{Biochemical Variables}

Nonfasting blood samples were obtained using venipuncture between 9:00 a.m. and 2:00 p.m. Processing, aliquoting, and freezing were performed at the Core Genetics Laboratory of The Johns Hopkins University School of Medicine following a standardized protocol. Serum carotenoids were determined using high-performance liquid chromatography in the laboratory of one of the investigators (RDS). Serum carotenoids were calculated as the sum of $\alpha$-carotene, $\beta$-carotene, $\beta$-cryptoxanthin, lutein/zeaxanthin, and lycopene in $\mathrm{mmol} / \mathrm{L}$. Low serum carotenoids were defined as the lowest tertile of carotenoid level. Quest Diagnostics Laboratories (Teterboro, $\mathrm{NJ}$ ) performed the other serum biochemical measurements. Total cholesterol, highdensity lipoprotein cholesterol, triglycerides, glycosylated hemoglobin and albumin, and low-sensitivity CRP were sent the same day to Quest Diagnostic Laboratories after processing. High CRP was defined as the highest tertile of CRP level.

\section{Analytical Plan}

First, the characteristics of the women in the study sample are described, including demographic characteristics, diseases 
and conditions, biochemical characteristics, individual frailty indicators, and frailty status according to three WHO BMI categories ${ }^{25,26}$ (underweight group was dropped). Crude frequencies and means \pm standard deviations were calculated; statistical tests for trend were performed using logistic regression for categorical variables (race, education, clinical conditions, frailty indicators) and ordinary least-square regression for continuous variables (age, BMI, biochemical variables). The variable for the evaluation of serum carotenoids was log-transformed to correct for skewed distribution. Frequency analysis also described the pattern of frailty criteria of women in the BMI categories that resulted in their being classified as frail. Frequency analysis was also used to describe the baseline relationship between the frailty syndrome and demographic and clinical characteristics of the participating women. Age-adjusted logistic regression models were used to determine whether there was a statistically significant trend in the increasing proportions of women with a given characteristic (e.g., low education) who were nonfrail, prefrail, and frail.

Multinomial logistic regression ${ }^{28}$ was used to study the cross-sectional association between obesity and the frailty syndrome, allowing the modeling of the prefrail and frail states (reference group $=$ not frail). A series of multinomial logistic regression models were sequentially adjusted for all variables hypothesized as potential confounders of the association between the frailty syndrome and obesity or suggested by available literature. ${ }^{3,29}$ The covariate of interest, BMI, was analyzed in categories, using BMI of $25 \mathrm{~kg} / \mathrm{m}^{2}$ to less than $30 \mathrm{~kg} / \mathrm{m}^{2}$ as the reference. Other covariates included age, low education, race, comorbid conditions, and biochemical indicators of inflammatory and antioxidant status. Comorbid conditions chosen were those associated with the frailty syndrome in the CHS cohort: diabetes mellitus, coronary artery disease (a composite variable considered present if angina pectoris or myocardial infarction was present), congestive heart failure (CHF), peripheral vascular occlusive disease (PVOD), chronic obstructive pulmonary disease and osteoarthritis. Many of these conditions are also related to obesity, for example, osteoarthritis and diabetes mellitus, and can potentially confound or mediate the relationship between obesity and frailty. Finally, low CRP and low serum carotenoids were added to the model. Low CRP was added because of the association between obesity and inflammation ${ }^{14}$ and between inflammation and frailty. ${ }^{30}$ Serum carotenoids were added because recent research has demonstrated the relationship between low antioxidant levels and frailty. ${ }^{15}$ The rationale for the three sequential models is that the first model includes potential demographic confounders of the relationship between obesity and frailty, the second model adds the comorbid conditions, and the third model adds the biochemical indicators of inflammation and antioxidant levels. Thus, Models 2 and 3 allow the hypothesis to be tested that comorbid conditions (Model 2) or inflammatory or antioxidant status (Model 3), because they are confounders or mediators of the relationship between obesity and frailty, account for the apparent crude association found in descriptive analyses.

To appropriately interpret inferences derived from the combined data back to the sampling population of com- munity-dwelling women, aged 70 to 79 , study-specific probability weights were used for all analyses. Construction of the weights has been detailed previously. ${ }^{19}$ Probability weights were incorporated into all the descriptive and regression analyses. Statistical programs used were SAS, version 8 (SAS Institute, Inc., Cary, NC) and Stata, version 8 (Stata Corp., College Station, TX).

\section{RESULTS}

Table 2 shows the characteristics of the women according to their BMI category. Some characteristics, as expected, showed a consistent increasing trend from lowest BMI category analyzed ( 18.5 to $<25 \mathrm{~kg} / \mathrm{m}^{2}$ (as discussed, women with BMI $<18.5 \mathrm{~kg} / \mathrm{m}^{2}$ were excluded)) to highest (obese, $\geq 30 \mathrm{~kg} / \mathrm{m}^{2}$ ), including diabetes mellitus, osteoarthritis of the knees, PVOD, and difficulty with activities of daily living and instrumental activities of daily living. CRP and triglyceride levels increased with increasing BMI; serum carotenoid and high-density lipoprotein cholesterol levels decreased. Frailty phenotype indicators of exhaustion and low physical activity had greater frequencies at low and high BMI levels. Weight loss decreased as BMI increased, whereas the prevalence of slowness and weakness increased with increasing BMI category.

Figure 1 illustrates the association between BMI category with being nonfrail, prefrail, and frail. The overall prevalence of frailty in the sample was $8 \%$; with $43 \%$ of women having prefrail status and $49 \%$ being nonfrail. The proportion of women who were nonfrail decreased with increasing BMI, the proportion of women who were prefrail increased with increasing BMI, and the proportion of women who were frail was lowest for women with a BMI of $25 \mathrm{~kg} / \mathrm{m}^{2}$ to less than $30 \mathrm{~kg} / \mathrm{m}^{2}$ and highest for women with a BMI of $30 \mathrm{~kg} / \mathrm{m}^{2}$ and higher.

The pattern of frailty criteria was examined in each of the BMI categories. For BMI of $18.5 \mathrm{~kg} / \mathrm{m}^{2}$ to less than $25 \mathrm{~kg} / \mathrm{m}^{2}$, the most common criteria pattern was low activity, weakness, slowness, and weight loss. Low activity, weakness, and slowness was the most common criteria pattern for the other two BMI categories. The second most common criteria pattern for all BMI groups was exhaustion, low activity, and slowness.

Table 3 shows the univariate association between frailty and other covariates hypothesized or suggested by the literature to be associated with frailty: low education, African-American race, comorbid conditions, high CRP, and low serum carotenoids. A significant and consistent trend for all covariates except race was that the proportion of women with a condition increased going from nonfrail to prefrail to frail.

Table 4 shows the results of sequential multinomial logistic regression models investigating the association between obesity and prefrail and frail (reference group was the nonfrail group), adjusting for covariates. In Model 3, obesity was significantly associated with prefrail and frail status, even after adjustment for all covariates considered. BMI of $18.5 \mathrm{~kg} / \mathrm{m}^{2}$ to less than $25 \mathrm{~kg} / \mathrm{m}^{2}$ was not significantly associated with prefrail or frail status. (BMI of 25 to $<30 \mathrm{~kg} / \mathrm{m}^{2}$ was chosen as the reference group because it had the lowest proportion of frail women.) Age was significantly associated only with frailty; low education 
Table 2. Characteristics of Women Aged 70 to 79 by Body Mass Index (BMI): Women's Health and Aging Study I and II Combined

\begin{tabular}{|c|c|c|c|c|}
\hline \multirow[b]{2}{*}{ Characteristic } & \multirow[b]{2}{*}{ All } & \multicolumn{3}{|c|}{ BMI } \\
\hline & & $\begin{array}{c}18.5 \text { to } \\
<25 \mathrm{~kg} / \mathrm{m}^{2} \\
(\mathrm{n}=200)\end{array}$ & $\begin{array}{c}25 \text { to } \\
<30 \mathrm{~kg} / \mathrm{m}^{2} \\
(\mathrm{n}=224)\end{array}$ & $\begin{array}{c}\geq 30 \mathrm{~kg} / \mathrm{m}^{2} \\
(\mathrm{n}=175)\end{array}$ \\
\hline \multicolumn{5}{|l|}{ Demographic } \\
\hline Age, mean $\pm S D^{*}$ & $74.0 \pm 2.7$ & $74.4 \pm 2.7$ & $73.9 \pm 2.8$ & $73.7 \pm 2.6$ \\
\hline African American, \% & 20.3 & 13.8 & 14.5 & 32.9 \\
\hline Less than high school education, \%* & 22.6 & 14.2 & 19.3 & 34.4 \\
\hline \multicolumn{5}{|l|}{ Clinical } \\
\hline Body mass index, mean $\pm \mathrm{SD}^{*}$ & $27.9 \pm 5.6$ & $22.3 \pm 1.8$ & $27.5 \pm 1.5$ & $34.6 \pm 4.6$ \\
\hline Diabetes mellitus \%* & 14.0 & 9.3 & 11.7 & 20.4 \\
\hline Congestive heart failure, \% & 12.5 & 10.8 & 9.0 & 17.6 \\
\hline Chronic obstructive pulmonary disease, $\%$ & 38.3 & 45.5 & 35.8 & 33.7 \\
\hline Peripheral vascular occlusive disease, $\%^{\dagger}$ & 17.1 & 13.4 & 15.1 & 23.8 \\
\hline Coronary artery disease, \% & 27.3 & 29.2 & 26.2 & 27.7 \\
\hline Osteoarthritis of knees, \%* & 43.0 & 31.3 & 40.7 & 60.2 \\
\hline Difficulty with $\geq 1$ activities of daily living, $\% * \neq$ & 23.3 & 14.9 & 21.1 & 32.3 \\
\hline Difficulty with $\geq 1$ instrumental activities of daily living, $\% * \S$ & 14.8 & 9.0 & 11.2 & 22.2 \\
\hline \multicolumn{5}{|l|}{ Biochemical, mean \pm SD } \\
\hline C-reactive protein, $\mathrm{mg} / \mathrm{L}^{*}$ & $6.0 \pm 6.8$ & $4.9 \pm 6.3$ & $5.6 \pm 6.1$ & $7.8 \pm 7.8$ \\
\hline Total cholesterol, mg/dL & $233.2 \pm 39.1$ & $232.0 \pm 41.2$ & $235.8 \pm 38.1$ & $233.2 \pm 39.2$ \\
\hline High-density lipoprotein, mg/dL* & $55.5 \pm 15.9$ & $59.8 \pm 18.0$ & $55.3 \pm 14.7$ & $50.6 \pm 13.3$ \\
\hline Triglycerides, mg/dL* & $164.8 \pm 97.5$ & $149.9 \pm 89.9$ & $168.9 \pm 98.9$ & $176.0 \pm 99.1$ \\
\hline Albumin, $\mathrm{g} / \mathrm{L}$ & $4.2 \pm 0.3$ & $4.2 \pm 0.3$ & $4.2 \pm 0.3$ & $4.1 \pm 0.3$ \\
\hline Total carotenoids, $\mathrm{mmol} / \mathrm{L}^{*}$ & $0.52 \pm 0.50$ & $0.62 \pm 0.52$ & $0.53 \pm 0.48$ & $0.41 \pm 0.46$ \\
\hline \multicolumn{5}{|l|}{ Frailty indicators, \% } \\
\hline Self-reported weight loss* & 84 & 12.1 & 5.5 & 5.2 \\
\hline Self-reported exhaustion & 13.2 & 14.6 & 9.0 & 13.9 \\
\hline Weakness, low grip strength * & 19.8 & 10.4 & 16.2 & 31.0 \\
\hline Slow walking speed* & 32.2 & 17.5 & 22.7 & 47.4 \\
\hline Decreased activity level, kcall & 22.0 & 16.0 & 14.2 & 29.3 \\
\hline
\end{tabular}

${ }^{*} P<.001 ;{ }^{\dagger} P<.01$.

${ }^{\ddagger}$ Transferring, bathing, dressing, eating, using the toilet.

${ }^{\S}$ Preparing meals, shopping, using the telephone, doing light housework.

"Less than $90 \mathrm{kcal}$ expenditure of physical activity per week.

was significantly associated with prefrailty and frailty. Of the comorbid conditions considered, diabetes mellitus, PVOD, and CHF were significantly associated with prefrailty and frailty; coronary artery disease with prefrailty only; and chronic obstructive pulmonary disease and osteoarthritis with frailty only. CRP was not associated with either, and low carotenoid level was associated with frailty only.

\section{DISCUSSION}

This research demonstrates that obesity is associated with a described frailty phenotype in multiple ways, consistent with the first hypothesis. High BMI and obesity are associated with several frailty phenotype indicators. High BMI is also associated with increasing likelihood of a prefrail state, although overweight status (BMI of 25 to $<30 \mathrm{~kg} /$ $\mathrm{m}^{2}$ ) is least associated with frailty. Obesity (BMI of $\geq 30 \mathrm{~kg} /$ $\mathrm{m}^{2}$ ) is associated with full-blown clinical frailty, and this association is not decreased when demographic character- istics, comorbidities, increased inflammation, or low antioxidant capacity are considered.

Frailty has often been considered a wasting disorder, but data from the CHS have also linked frailty to higher BMI. ${ }^{16}$ The current study focuses on the association between the CHS-described frailty phenotype and increasing BMI; therefore, underweight women were not studied. However, after the obese group, the normal BMI group included the second highest percentage of frail women. The most common pattern for the normal BMI group was four indicators - the same pattern as for the other two BMI groups, with the addition of the weight loss criterion. Similar patterns of criteria leading to the frailty assignment in all BMI groups suggests that the hypothesis that mechanisms leading to frailty may be more common in those with high BMI but may not depend on BMI. Weight loss may be an exception to this because it is an important indicator in lower-weight women but was not reported by higherweight women.

Although an association between obesity and frailty appears to be present, the biomechanical burden of fat in 


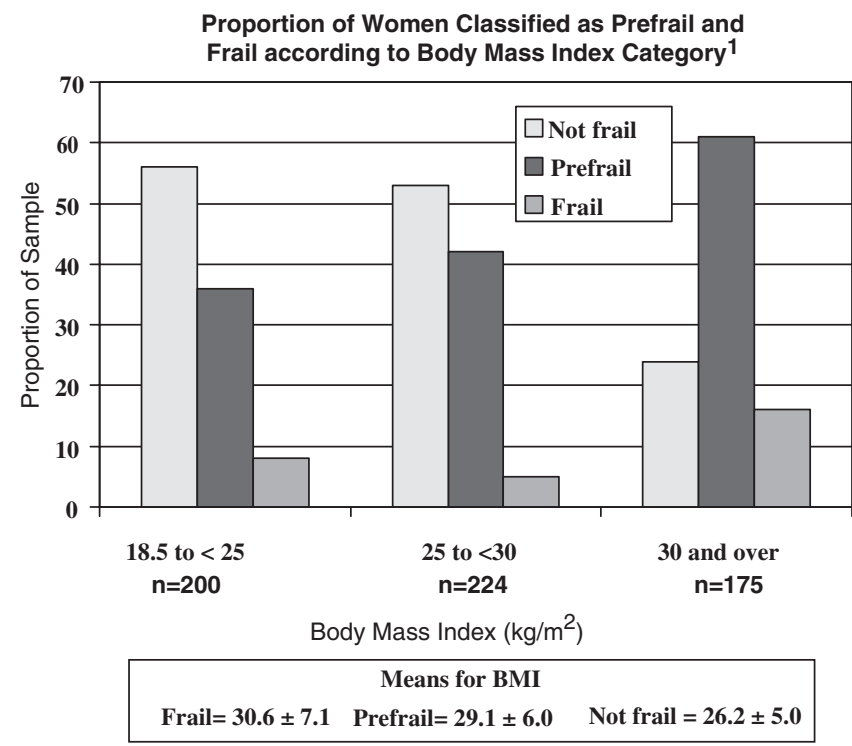

Figure 1. This figure shows the association between body mass index (BMI) and being nonfrail, prefrail, and frail. Nonfrail was associated with lower BMI, and prefrail was associated with higher BMI. The lower and higher BMI groups had a larger percentage of frail women than the middle groups.

overweight and obese people could confound this finding. However, a substantial amount of current research has demonstrated that obesity is associated with several physiological markers that have also been associated with frailty, most notably increased levels of inflammatory markers ${ }^{16,30}$ and low antioxidant capacity. ${ }^{32}$ These data showed a significant crude association between high CRP and low carotenoids and high BMI. However, high CRP was not associated with prefrail or frail status in a multivariate model that included BMI and comorbid diseases as covariates. These findings give physiological plausibility to the frailty-obesity association, but with these cross-sectional data, it is not possible to ascribe a causal association between obesity and frailty. Longitudinal information and further pathophysiological study are needed to sort out causation.

Research concerning "sarcopenic obesity" provides a key insight into the association between frailty and obesity. A syndrome has been described in older adults defined by a mismatch of fat mass to muscle mass. ${ }^{10,11}$ They have demonstrated a convincing association between disability, particularly mobility and strength-based disabilities, in older adults and sarcopenic obesity. Sarcopenic obesity has not yet been associated with inflammatory dysregulation, which might be hypothesized, given the association between increased inflammation and increased fat and decreased muscle mass. No data yet available link sarcopenic obesity with the syndrome of frailty. These data do not include body composition or information regarding regional fat deposition. It is possible that characteristics such as ratio of fat mass to muscle mass or amount of visceral versus peripheral fat are more strongly related to the presence or absence of the frailty syndrome than just BMI.

In addition to its association with obesity, this research demonstrated that frailty was associated with diabetes mellitus, CHF, PVOD, and osteoarthritis. Coronary artery disease, although significantly associated with prefrail status, was not associated with frail status in the WHAS sample. Because obesity is also associated with diabetes mellitus, atherosclerotic diseases, and osteoarthritis, it was hypothesized that these diseases would confound the association between the frailty syndrome and obesity, but contrary to this hypothesis, obesity was related independently to prefrail and frail status, even after controlling for comorbid chronic diseases.

This research has several limitations. Some important variables are not available, including variables about nutritional intake; body composition, including muscle and

Table 3. Baseline Association of Demographic and Health Characteristics with Frailty

\begin{tabular}{|c|c|c|c|c|}
\hline \multirow[b]{2}{*}{ Characteristic } & $\begin{array}{l}\text { Not Frail } \\
(n=293)\end{array}$ & $\begin{array}{l}\text { Prefrail } \\
(\mathrm{n}=258)\end{array}$ & $\begin{array}{c}\text { Frail } \\
(n=48)\end{array}$ & \multirow[b]{2}{*}{$P$-value } \\
\hline & \multicolumn{3}{|c|}{$\%$} & \\
\hline Low education & 11.66 & 28.55 & 45.76 & $<.001$ \\
\hline African American & 15.66 & 23.31 & 28.44 & 0.003 \\
\hline Diabetes mellitus & 7.11 & 18.52 & 27.74 & $<.001$ \\
\hline Peripheral vascular occlusive disease & 8.62 & 22.66 & 35.93 & $<.001$ \\
\hline Coronary artery disease & 18.16 & 35.12 & 37.46 & $<.001$ \\
\hline Congestive heart failure & 4.68 & 15.57 & 40.70 & $<.001$ \\
\hline Chronic obstructive pulmonary disease & 33.77 & 40.40 & 55.43 & .004 \\
\hline Osteoarthritis of knees & 34.47 & 50.20 & 56.07 & $<.001$ \\
\hline High CRP* & 30.91 & 38.81 & 46.21 & .01 \\
\hline Low serum carotenoids ${ }^{\dagger}$ & 25.25 & 40.33 & 50.99 & $<.001$ \\
\hline Difficulty with $\geq 1$ activity of daily living tasks $\ddagger$ & 7.47 & 33.34 & 63.30 & $<.001$ \\
\hline Difficulty with $>1$ instrumental activity of daily living tasks $\S$ & 3.97 & 20.18 & 48.34 & $<.001$ \\
\hline
\end{tabular}

\footnotetext{
${ }^{*}$ Highest tertile in high-sensitivity C-reactive protein $(\mathrm{CRP})(\mathrm{mg} / \mathrm{L})$.

${ }^{\dagger}$ Lowest tertile in sum of total carotenoids $(\mathrm{mmol} / \mathrm{L})$.

${ }^{\ddagger}$ Transferring, bathing, dressing, eating, using the toilet.

${ }^{\S}$ Preparing meals, shopping, using the telephone, doing light housework.
} 


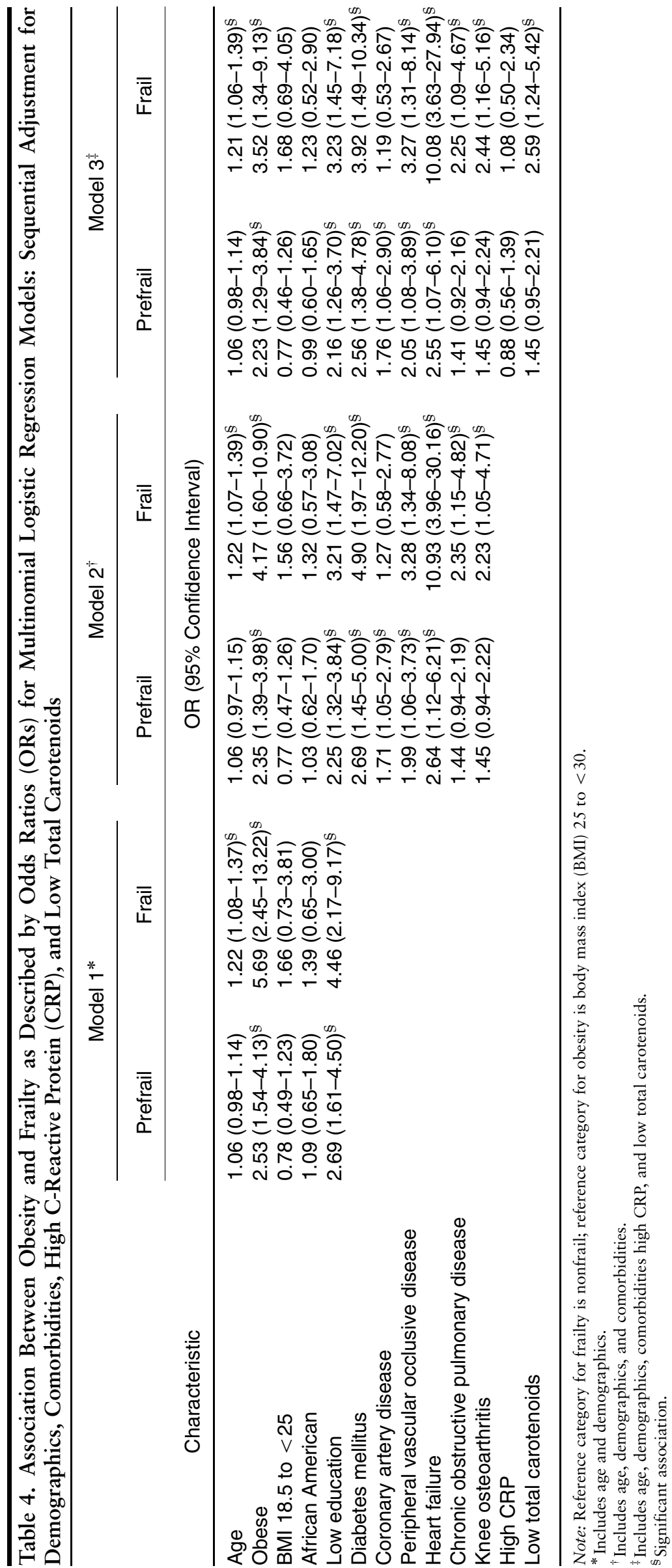


fat mass; and intentional/unintentional weight loss. The measure of frailty confounded the measures of low weight and physical activity and thus cannot be satisfactorily investigated. In addition, the cross-sectional study design cannot answer important questions about the association between obesity and frailty. Longitudinal data are critical to understanding the relationship between weight loss - unintentional and intentional-and frailty in obese older adults. Similarly, the contribution of physical activity level cannot be related to weight changes or frailty. For example, it is not possible to disentangle whether some obese people become frail from whether frail people have low activity and gain weight.

Another important limitation of this study is that using an all-female sample does not allow generalizability of the current findings to men. In addition, frailty is a relatively uncommon condition, so despite use of data from a large, population-based study, the sample size available for the study of several important associations is small. However, few larger studies are available that are population based and also have the variables available to study relationships between frailty and comorbidities.

This research has demonstrated a clear relationship between obesity and frailty. It also suggests that people with higher weight and obesity are more likely to be prefrail. This finding is consistent with results from the CHS, ${ }^{3}$ which demonstrated that prefrail people have a markedly greater risk than those who are not frail of becoming frail. Although frailty is not yet defined clinically, the finding of a high prevalence of prefrailty in older populations suggests an underlying risk of poor outcomes in some older people that can potentially be favorably modified. Clinically oriented prospective studies of frailty are needed to evaluate the importance of diagnosing prefrailty or frailty and to study potential interventions to decrease risks and poor health outcomes.

If the increasing levels of obesity in American adults lead to greater numbers of older obese people, these older obese people may have greater risk for frailty in addition to their greater risks of obesity-related diseases and disabilities. To better understand the association between obesity and frailty and whether it constitutes another adverse outcome of obesity, future prospective research is critical. In addition, given the difficulty of disentangling some of the key variables, research involving exercise interventions may be needed. However, as obesity rates increase in middle-aged and older adults, a better understanding of obesity and frailty, as well as the roles of physical activity and weight loss, may be important to improve the health of older adults.

\section{ACKNOWLEDGMENTS}

The authors thank Karen Bandeen-Roche for her assistance relating WHAS frailty criteria to CHS frailty criteria, and Tisha Moore for her manuscript preparation and assistance with development of figures and tables.

\section{REFERENCES}

1. Morley JE, Perry HM 3rd, Miller DK. Editorial: Something about frailty. J Gerontol A Biol Sci Med Sci 2002;57A:M698-M704.

2. Lipsitz LA. Dynamics of stability. The physiologic basis of functional health and frailty. J Gerontol A Biol Sci Med Sci 2002;57A:B115-B125.
3. Fried LP, Tangen CM, Walston J et al. Frailty in older adults: Evidence for a phenotype. J Gerontol A Biol Sci Med Sci 2001;56A:M146-M156.

4. Hogan DB, MacKnight C, Bergman H. Models, definitions, and criteria of frailty. Aging Clin Exp Res 2003;15:1-29.

5. Lunney JR, Lynn J, Hogan C. Profiles of older medicare decedents. J Am Geriatr Soc 2002;50:1108-1112.

6. Isaacs B, Gunn J, McKechan A et al. The concept of pre-death. Lancet 1971;1:1115-1118.

7. Visscher TL, Seidell JC. The public health impact of obesity. Annu Rev Public Health 2001;22:355-375.

8. Roubenoff R. The pathophysiology of wasting in the elderly. J Nutr 1999;129:256S-259S.

9. Roubenoff R. Sarcopenia: A major modifiable cause of frailty in the elderly. J Nutr Health Aging 2000;4:140-142.

10. Baumgartner RN, Koehler KM, Gallagher D et al. Epidemiology of sarcopenia among the elderly in New Mexico. Am J Epidemiol 1998;147:755-763.

11. Baumgartner RN. Body composition in healthy aging. Ann N Y Acad Sci 2000;904:437-448.

12. Roubenoff R, Hughes VA. Sarcopenia: Current concepts. J Gerontol A Biol Sci Med Sci 2000;55A:M716-M724.

13. Roubenoff R. Sarcopenic obesity. Does muscle loss cause fat gain? Lessons from rheumatoid arthritis and osteoarthritis. Ann N Y Acad Sci 2000;904: 553-557.

14. Visser M, Bouter LM, McQuillan GM et al. Elevated C-reactive protein levels in overweight and obese adults. JAMA 1999;282:2131-2135.

15. Semba RD, Blaum CS, Guralnik JM et al. Carotenoid and vitamin E status are associated with indicators of sarcopenia among older women living in the community. Aging Clin Exp Res 2003;15:482-487.

16. Walston J, McBurnie MA, Newman A et al. Frailty and activation of the inflammation and coagulation systems with and without clinical comorbidities. Arch Intern Med 2002;162:2333-2341.

17. Newman AB, Gottdiener JS, McBurnie MA et al. Associations of subclinical cardiovascular disease with frailty. J Gerontol A Biol Sci Med Sci 2001;56A: M158-M166.

18. Ferrucci L, Guralnik JM, Bandeen-Roche KJ et al. Physical performance measures. In: Guralnik J, Fried LP, Simonsick EM et al. The Women's Health and Aging Study: Health and Social Characteristics of Older Women with Disability. Bethesda, MD: National Institute on Aging, 1995, pp 35-49.

19. Guralnik J, Fried LP, Simonsick EM et al. Screening the community-dwelling population for disability. In: Guralnik J, Fried LP, Simonsick EM et al. The Women's Health and Aging Study: Health and Social Characteristics of Older Women with Disability. Bethesda, MD: National Institute on Aging, 1995, pp 9-18.

20. Fried LP, Bandeen-Roche K, Chaves PH et al. Preclinical mobility disability predicts incident mobility disability in older women. J Gerontol A Biol Sci Med Sci 2000;55A:M43-M52.

21. Guralnik J, Fried L, Simonsick E et al. The Women's Health and Aging Study: Health and Social Characteristics of Older Women with Disability (NIH Publication no. 95-4009). Bethesda, MD: National Institutes of Health, 1995.

22. Cappola AR, Bandeen-Roche K, Wand GS et al. Association of IGF-I levels with muscle strength and mobility in older women. J Clin Endocrinol Metab 2001;86:4139-4146.

23. Siscovick DS, Fried L, Mittelmark $M$ et al. Exercise intensity and subclinical cardiovascular disease in the elderly. The Cardiovascular Health Study. Am J Epidemiol 1997;145:977-986.

24. Simonsick EM, Phillips C, Skinner E et al. The daily lives of disabled older women. In: Guralnik J, Fried LP, Simonsick EM et al. The Women's Health and Aging Study: Health and Social Characteristics of Older Women with Disability. Bethesda, MD: National Institute on Aging, 1995, pp 50-69.

25. Obesity: Preventing and Managing the Global Epidemic. Report of a WHO Consultation on Obesity. Geneva, Switzerland: World Health Organization, 2000.

26. Physical Status: The Use and Interpretation of Anthropometry. Geneva, Switzerland: World Health Organization, 1995

27. Fried LP, Kasper J, Williamson J et al. Disease ascertainment algorithms. In: Guralnik J, Fried LP, Simonsick EM et al. The Women's Health and Aging Study: Health and Social Characteristics of Older Women with Disability. Bethesda, MD: National Institute on Aging, 1995, Appendix E.

28. Hosmer D, Lemeshov S. Applied Logistic Regression. New York: John Wiley and Sons, 1989.

29. Strawbridge WJ, Shema SJ, Balfour JL et al. Antecedents of frailty over three decades in an older cohort. J Gerontol B Psychol Sci Soc Sci 1998;53B:S9-S16.

30. Ershler WB, Keller ET. Age-associated increased interleukin- 6 gene expression, late-life diseases, and frailty. Annu Rev Med 2000;51:245-270.

31. Bortz WM, 2nd. A conceptual framework of frailty: A review. J Gerontol A Biol Sci Med Sci 2002;57A:M283-M288. 